\title{
Non-invasive brain stimulation applied to Heschl's gyrus modulates pitch discrimination
}

\section{Christoph Mathys, Psyche Loui, Xin Zheng and Gottfried Schlaug*}

Music and Neuroimaging Laboratory, Department of Neurology, Beth Israel Deaconess Medical Center and Harvard Medical School, Boston, MA, USA

\section{Edited by:}

Eckart Altenmüller, University of Music and Drama Hannover, Germany

\section{Reviewed by:}

Lutz Jäncke, University of Zurich, Switzerland

Christo Pantev, University of Muenster, Germany

Eckart Altenmüller, University of Music and Drama Hannover, Germany

\section{*Correspondence:}

Gottfried Schlaug, Music and Neuroimaging Laboratory, Department of Neurology, Beth Israel Deaconess Medical Center and Harvard Medical School, 330 Brookline Avenue, Boston, MA 02215, USA.

e-mail: gschlaug@bidmc.harvard.edu
The neural basis of the human brain's ability to discriminate pitch has been investigated by functional neuroimaging and the study of lesioned brains, indicating the critical importance of right and left Heschl's gyrus (HG) in pitch perception. Nonetheless, there remains some uncertainty with regard to localization and lateralization of pitch discrimination, partly because neuroimaging results do not allow us to draw inferences about the causality. To address the problem of causality in pitch discrimination functions, we used transcranial direct current stimulation to downregulate (via cathodal stimulation) and upregulate (via anodal stimulation) excitability in either left or right auditory cortex and measured the effect on performance in a pitch discrimination task in comparison with sham stimulation. Cathodal stimulation of $\mathrm{HG}$ on the left and on the right hemispheres adversely affected pitch discrimination in comparison to sham stimulation, with the effect on the right being significantly stronger than on the left. Anodal stimulation on either side had no effect on performance in comparison to sham. Our results indicate that both left and right $\mathrm{HG}$ are causally involved in pitch discrimination, although the right auditory cortex might be a stronger contributor.

Keywords: pitch perception, brain stimulation, transcranial direct current stimulation, auditory cortex

\section{INTRODUCTION}

Functional brain mapping studies and analysis of human lesion studies have been used to understand the neural correlates and hemispheric dominance of pitch perception (Zatorre et al., 1994; Platel et al., 1997; Griffiths et al., 1999; Schuppert and Altenmuller, 1999; Schuppert et al., 2000; Gaab and Schlaug, 2003; Gaab et al., 2003, 2006; Hyde et al., 2008). While lesion and imaging studies have yielded interesting results, they both have disadvantages: lesion studies by definition look at non-normal brains; lesions are usually not limited to a particular brain region that subserves a single function; and in most cases the same brain has not been investigated in both lesioned and non-lesioned states. On the other hand, functional imaging studies have an inherent problem, since neuroimaging studies mainly offer correlations between brain activation and behavior and it is difficult to make causal inferences. Analysis of patients with focal brain lesions and functional imaging studies seem to indicate a critical role of the right, but also the left HG, possibly to a lesser degree than the right HG, in pitch discrimination (Zatorre, 1988; Peretz, 1990; Johnsrude et al., 2000; Patterson et al., 2002; Tramo et al., 2005). Some people have argued that the right HG might be the more important region in pitch discrimination since the right hemisphere specializes in spectral perception whereas the left hemisphere specializes in temporal perception (Zatorre et al., 2002; Hickok and Poeppel, 2007; Okamoto et al., 2009). Scott and Wise (2004) argue that the left hemisphere's preference for stimuli rich in temporal structural details (such as they occur in speech), as opposed to the right hemisphere's preference for stimuli with melodic pitch information, might not be rooted in a better ability to process such stimuli, but in a bias for extracting meaning, which may have its developmental origins in language acquisition. Hemispheric specializations for pitch perception and pitch memory have also been seen in musically trained individuals (Gaab and Schlaug, 2003) and in musicians with absolute pitch compared to musicians without absolute pitch (Ohnishi et al., 2001; Schulze et al., 2009; Loui et al., 2010).

An alternative method to examine the causal contributions of a brain region to a particular behavior is to use non-invasive brain stimulation to create a temporary "virtual lesion." Transcranial magnetic stimulation (TMS) and transcranial direct current stimulation (tDCS) are two such non-invasive methods that have been used to examine causal relationships between brain and behavior (Chen et al., 1997; Vines et al., 2006a,b). Compared to tDCS, TMS has two disadvantages in auditory studies: firstly, TMS emits loud clicking sounds during stimulation, which may introduce confounding effects on the auditory cortex. Secondly, when applied around the ear, TMS affects the temporalis muscle, which may contribute to local pain or headaches more than TMS applied to other sites (e.g., prefrontal cortex or motor cortex). In contrast, tDCS is not associated with any sound. It is not known to affect any peri-aural muscles and is typically well tolerated by the subject, mostly causing only a local tingling or itching sensation in the first few minutes after the stimulation is turned on. tDCS uses a weak direct current that flows between two cephalic electrodes to modulate levels of regional brain excitability. It enables us to test the effects of upregulating and downregulating neuronal excitability (depending on which way the current flows between the two electrodes) on behavior if the tested behavior draws on the region whose activity is being modulated (Nitsche and Paulus, 2000, 2001; Liebetanz et al., 2002; Siebner et al., 2004; Vines et al., 2006a,b, 2008). Cathodal stimulation (i.e., downregulating excitability) can be seen as similar to creating a temporary dysfunction ("virtual lesion") in the brain region underlying the electrode 
location. Anodal stimulation, on the other hand, is shown to lead to enhanced performance due to increased excitability of the stimulated neural tissue (Nitsche and Paulus, 2000). The stimulation effect is only temporary with behavioral/cognitive effects lasting for about $30 \mathrm{~min}$ after a 20 -min stimulation.

As a test of pitch discrimination ability, we used the established psychophysical staircase procedure of an adaptive two-interval twoalternative forced choice pitch direction discrimination task. We presented subjects with two pure sine-wave tones and the subjects had to decide whether the second tone was higher or lower than the first. Pitch direction discrimination differs from pitch difference discrimination in that it involves a higher/lower judgment and not simply a same/different judgment. There are indications that pitch direction discrimination ability is more markedly right-lateralized than same/different judgments of pitch (Johnsrude et al., 2000; Tramo et al., 2005). In addition to its rightward laterality, pitch direction discrimination has been shown to be sensitive to effects of learning over the course of multiple testing sessions, especially for individuals with little to no musical training (Micheyl et al., 2006). On the other hand, musically trained subjects (and occasionally even musically untrained subjects) may perform fairly stably in pitch direction discrimination at the outset, but may occasionally habituate and even regress in performance over the course of prolonged testing (Micheyl et al., 2006). These learning/habituation effects represent necessary intrasubject variability over the course of testing and are accounted for as covariates in the present statistical design.

Our hypothesis was that cathodal stimulation over the right more so than cathodal stimulation over the left HG would lead to a decrement in performance accuracy in a pitch discrimination task. Furthermore, we hypothesized that anodal stimulation would lead to an increment in performance accuracy, while sham stimulation would not lead to any significant change in performance accuracy.

\section{MATERIALS AND METHODS SUBJECTS}

Three groups of subjects participated in the study. Subjects were randomly assigned to the anodal and cathodal groups. Subjects randomized to the cathodal group ( $n=11 ; 5$ females; mean age: $28.9 \pm 9.2$ years) received $\mathrm{tDCS}$ with the cathodal electrode over the skull position of the first transverse gyrus of Heschl (HG), while subjects randomized to the anodal group ( $n=11 ; 6$ females; mean age: $24.7 \pm 3.9$ years) received tDCS with the anodal electrode over the same location. One subject was excluded from the analysis of the cathodal group after he showed high variability and nonreproducible performance on the pre-test assessments; thus all the statistical analyses and the results are based on 10 subjects in the cathodal group. The reference electrode for both conditions was over the contralateral supraorbital region. In order to gain additional confidence in the results found for the cathodal group, a third group of subjects ( 2 men and 2 women; mean age $24.0 \pm 1.4$ years) was added later as a control group who received cathodal stimulation over the occipital lobe. The stimulation over the occipital lobe was done to control for the possibility that any brain stimulation might have an effect on pitch discrimination. This group will be referred to as the "cathodal-control" group. All subjects were right-handed as assessed by the Edinburgh Handedness Inventory (Oldfield, 1971) and had no history of neurological, psychiatric, or auditory problems. All of our subjects had some musical background (e.g., regularly playing a musical instrument or singing): subjects in the cathodal, anodal, and cathodal-control groups had a median (interquartile range in brackets) of 14.5 (8.3), 12.0 (9.5), and 14.5 (3.5) years of musical experience respectively, but there was no significant difference between the three groups. This study was approved by the Institutional Review Board of Beth Israel Deaconess Medical Center, and all subjects gave written informed consent.

\section{TASK}

Subjects performed the psychophysical staircase procedure of an adaptive two-interval two-alternative forced-choice pitch direction discrimination task. They were seated in front of a laptop computer with headphones and a mouse. In each trial they heard a series of two pure tones presented over the headphones at approximately $70 \mathrm{~dB}$. Each pure tone had a 500-ms duration, with rise and fall times of $40 \mathrm{~ms}$ each. A silent interval of $500 \mathrm{~ms}$ was presented between the tones. The first tone was fixed at $200 \mathrm{~Hz}$ (center frequency) whereas the second tone was randomly either higher or lower than the first in frequency, with frequency differences to be described below. Subjects had to decide whether the second tone was higher or lower than the first, and to indicate their response by clicking the right or left mouse button, respectively. They were told to make decisions as quickly as possible and reaction time was recorded. The next trial followed $1000 \mathrm{~ms}$ after the button press.

The center frequency of $200 \mathrm{~Hz}$ was chosen because tonotopic mappings indicate that lower frequencies are more laterally localized within Heschl's Gyrus compared to higher frequencies which are typically more mesial (Pantev et al., 1989; Formisano et al., 2003; Talavage et al., 2004; Langers et al., 2007). A function that is more laterally localized in Heschl's Gyrus is more likely to be modulated by, and hence susceptible to transcranially applied non-invasive brain polarization with $\mathrm{tDCS}$.

The task started with tones at an interval of 400 cents (level 1). Every series of three consecutive correct decisions led to a halving of the current interval, while every wrong decision led to its doubling. Subjects could thus progress (and fall back again) from level 1 (400 cents) to level 9 ( 1.5625 cents). Every time the direction of level change reversed from down to up or from up to down, the level at which this occurred was recorded as a turnaround point. One run of the task was completed once a subject had gone through eight turnaround points. After a resting period of $15 \mathrm{~s}$ the task automatically resumed with the next run until eight runs were completed. A total of 64 turnaround point intervals were thus acquired in approximately $20 \mathrm{~min}$.

\section{PROCEDURE}

In a practice session without tDCS, subjects completed the task twice. This allowed us to make sure their performance was sufficiently stable to include them in the study. At least 1 day after this, for the cathodal and anodal groups, three tDCS sessions took place in randomized order, each on a separate day and corresponding to one of three stimulation conditions. The three stimulation conditions were (1) stimulation over right HG ("right"), (2) stimulation over left HG ("left"), and (3) sham stimulation ("sham"). At the 
beginning of each experimental session, subjects did the task once to refamiliarize themselves with it. Thereafter, the actual or sham stimulation took place, following which the effect of this stimulation on pitch discrimination was assessed. During the stimulation period, a constant current stimulator (Phoresor II PM850; Iomed, Inc., Salt Lake City, UT, USA) delivered a current of $2.0 \mathrm{~mA}$ for $25 \mathrm{~min}$. The order of the stimulation conditions was randomized to neutralize any order effects. During sham stimulation, a brief stimulation was delivered for under $1 \mathrm{~min}$ and then gradually ramped down to $0 \mathrm{~mA}$, such that in both real and sham stimulation conditions the subjects felt the tingling sensation of stimulation, but in the sham stimulation condition no current was delivered for the duration of the "stimulation" session. This made it highly unlikely that the subjects could have been aware whether each session was "real" vs. "sham."

The active electrode ( area $=16.3 \mathrm{~cm}^{2}$ ) was placed $1 \mathrm{~cm}$ inferior to the halfway point between C3 and T3 of the 10-20 system of EEG electrode placement for stimulation of left HG. For stimulation of right $\mathrm{HG}$, the active electrode was correspondingly placed $1 \mathrm{~cm}$ inferior to the halfway point of $\mathrm{C} 4$ and T4. The correctness of this positioning was confirmed through anatomical MR images of several subjects. The position of MRI compatible markers at the electrode positions and the location of anatomical structures of interest is shown in Figure 1. The reference electrode $\left(\right.$ area $\left.=30 \mathrm{~cm}^{2}\right)$ was placed contralaterally to the active HG electrode in the supraorbital region, just above the eyebrow. The sham stimulation counterbalanced, with half of the subjects receiving sham stimulation on the left side, and the other half on the right side.

In the cathodal-control group, the active electrode was placed over the occipital lobe halfway between $\mathrm{O} 1$ and $\mathrm{O} 2$, while the reference electrode was placed either on the left or the right supraorbital area in a randomized fashion. This was done to investigate whether
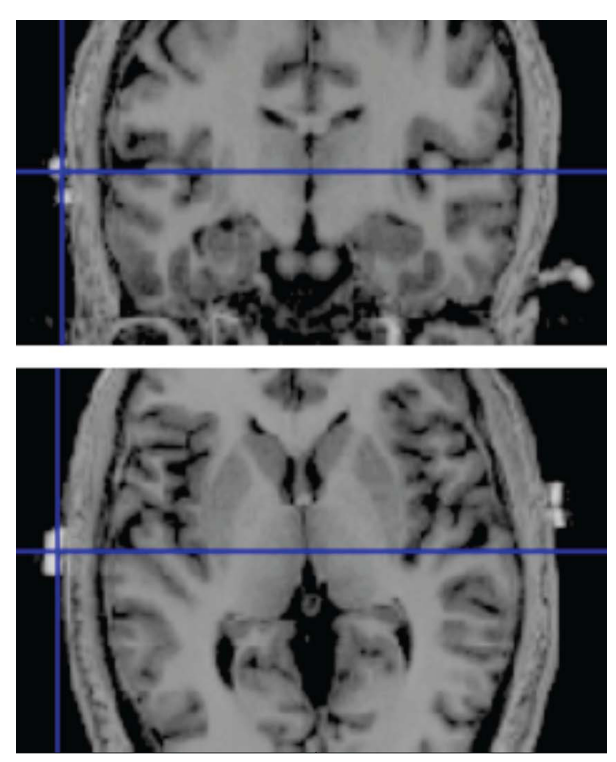

FIGURE 1 | High resolution T1-weighted image of a single subject with MRI compatible markers placed over the skull position of HG. Top: coronal section; bottom: axial section. The markers show a good correspondence between the electrode position and the presumed skull position of HG. cathodal stimulation alone, regardless of the placement of the active electrode, was responsible for any effects we saw in the cathodal group. The procedure was the same for this group as for the other experimental groups except that it only had two stimulation conditions: (1) occipital cathodal stimulation ("occipital”), and (2) sham stimulation ("sham"), whose order was randomized.

\section{DATA ANALYSIS}

The cent values (100 cents $=1$ semitone) of the 64 turnaround point intervals of the pitch discrimination task were logarithmically transformed because they were log-normally distributed.

To test the hypothesis that $\mathrm{tDCS}$ on $\mathrm{HG}$ influences pitch discrimination, we conducted an analysis of covariance (ANCOVA) for the cathodal and anodal groups with the factors of stimulation condition (containing three levels of left, right, and sham) and subject, and the covariate of learning/habituation. In addition to the main effects of stimulation condition, subject, and learning, the interaction between subject and learning was also included in the analysis. This reflected the idea that subjects would not only show a different baseline performance (i.e., the factor of subject) and a linear trend from session to session (i.e., the covariate of learning/habituation), but that subjects would also show individual differences in their learning effects. The interaction term was significant in both experimental (i.e., cathodal and anodal) groups and led to an improvement in Akaike's information criterion (AIC) in both groups, indicating an increase in the predictive power of the statistical model underlying the analysis of covariance. In the case where the stimulation condition factor was significant, treatment contrasts were assessed to determine the direction, size, and significance of the difference in performance between the different stimulation conditions. Where these differences were significant, effect sizes were determined by standardizing the regression coefficients against the residual standard error in the linear model underlying the analysis of covariance.

The interaction between learning and subject could not be included in the analysis of the cathodal-control group because in this case, due to there only being two stimulation conditions, the interaction is collinear to the stimulation condition. The main effect of the habituation/learning covariate proved significant for this group as well, and including it led to a better AIC value. In this group, not only the question whether stimulation had an effect on performance was of interest, but also whether the placement of the reference electrode on the left or right supraorbital area made any difference. The randomized, left or right, placement of the reference electrode (while the stimulation electrode was always in the same occipital location) allowed us to conduct an analysis of covariance with the factors "reference electrode condition" and "subject" while again correcting for a linear habituation/learning covariate. The factor describing the reference electrode condition had the levels "sham," "anodal, right," and "anodal, left." The covariate proved significant and again led to a better AIC value.

The three groups did not differ from each other at baseline. The baseline performances of the three groups were assessed with $t$-tests comparing subjects' baseline pitch direction discrimination thresholds. These thresholds were calculated as the geometric mean (i.e., the arithmetic mean on the logarithmic scale) of all turnaround point intervals of a subject in the sham condition and did not show any significant differences between groups. 


\section{RESULTS \\ CATHODAL GROUP}

Transcranial direct current stimulation produced a very highly significant effect in the cathodal group, as demonstrated by a significant main effect of stimulation condition $(p<0.0001)$ (Table 1 ). Subjects differed significantly in their baseline performance with each other, as demonstrated by the significant subject factor, but showed a significant main effect of learning/habituation. A significant interaction between subject and learning/habituation was found, suggesting that individuals differed significantly in their learning/habituation effects.

Testing our a priori contrasts revealed that cathodal stimulation over left as well as right $\mathrm{HG}$ interfered with pitch direction discrimination when compared with sham stimulation (left: $p<0.01$, right: $p<0.00001$; two-tailed). Furthermore, the effect of stimulation over right HG was significantly stronger than the effect of stimulation over the left HG ( $p<0.02$; two-tailed) (Table 2). The linear model underlying the analysis predicts that after correcting for

Table 1 |Analysis of covariance for the cathodal group.

\begin{tabular}{|c|c|c|c|c|}
\hline Source & Sum of squares & df & $F$-value & $p$-Value \\
\hline Stimulation condition & 16.37 & 2 & 11.364 & 0.0000124 \\
\hline Subject & 493.63 & 9 & 76.142 & $<2.2 \times 10^{-16}$ \\
\hline Learning/habituation & 8.65 & 1 & 12.007 & 0.0005419 \\
\hline $\begin{array}{l}\text { Subject } \times \text { learning/ } \\
\text { habituation }\end{array}$ & 73.40 & 9 & 11.322 & $<2.2 \times 10^{-16}$ \\
\hline Residuals & 1367.19 & 1898 & & \\
\hline
\end{tabular}

Response variable: natural logarithm of turnaround point interval in cents. df, degrees of freedom. All sources of variance are very highly significant, indicating that cathodal tDCS over HG had an influence on pitch direction discrimination. In addition to individual differences (the "subject" factor), there was an expected significant learning/habituation effect. The significant interaction between subject and learning/habituation means that the amount of learning/habituation differed significantly among subjects.

Table 2 | Contrasts of stimulation conditions in the cathodal group.

\begin{tabular}{llllll}
\hline Contrast & Effect size & $\begin{array}{l}\text { Standard } \\
\text { error }\end{array}$ & df & t-Value & $\boldsymbol{p}$-Value \\
\hline $\begin{array}{l}\text { Cathodal } \\
\text { right vs. sham }\end{array}$ & 0.397 & 0.083 & 1898 & 4.764 & 0.00000204 \\
$\begin{array}{l}\text { Cathodal } \\
\text { left vs. sham }\end{array}$ & 0.210 & 0.077 & 1898 & 2.713 & 0.00672 \\
$\begin{array}{l}\text { Cathodal right } \\
\text { vs. cathodal left }\end{array}$ & 0.187 & 0.077 & 1898 & 2.413 & 0.0159 \\
\end{tabular}

A priori treatment contrasts in the cathodal group were calculated after standardizing the response variable against the residual standard error of the analysis of covariance in Table 1. This yields contrast coefficients that are interpretable as effect sizes. The contrast of the "cathodal, right" vs. the "sham" condition indicates that $t D C S$ over right HG leads to a very highly significant deterioration in pitch direction discrimination performance amounting to $0.40( \pm 0.08)$ standard deviations ( \pm standard error). Stimulation on the left side leads to a highly significant deterioration of performance amounting to 0.2 ( \pm 0.08$)$ standard deviations ( \pm standard error). Finally, the contrast between stimulation on the left and on the right side shows that, compared to left-side stimulation as the baseline, right-side stimulation leads to a deterioration in performance of 0.19 ( \pm 0.08$)$ standard deviations ( \pm standard error). learning/habituation, the average subject from the cathodal group has a pitch direction discrimination threshold of 8.7 cents without stimulation, but one of 12.2 cents after cathodal stimulation over right $\mathrm{HG}$ and one of 10.4 cents after cathodal stimulation over left HG. The predicted threshold is the predicted value of the average turnaround point intervals in the pitch discrimination task. The main effects are shown in Figure 2 and individual subjects' results are shown in Figure 3.

A further question of interest was whether the effect of stimulation showed any variation with time after the end of stimulation. We investigated this for the cathodal and anodal groups by conducting analyses of variance within the subsets of data belonging to one stimulation condition. Within these subsets, we calculated the variance due to the factors "subject" and "run number." This last factor took values from " 1 " to " 8 " for the sequence of eight runs in each task session. Analyses were done for each stimulation condition ("right," "left," and "sham") with run number as an ordered factor for which polynomial contrasts were calculated. This allows us not only to detect linear trends but also to pick up any u-shaped temporal performance variations within sessions. Under none of the three stimulation conditions was there any significant variation of performance with time within sessions. This indicates that the effect of stimulation remained stable throughout the whole period of about $20 \mathrm{~min}$ it took the subjects to complete eight runs of the task.

\section{CATHODAL-CONTROL GROUP}

Two analyses of covariance were computed for this group. The first was designed to determine whether cathodal stimulation over the occipital lobe led to any significant effect on pitch discrimination performance compared to sham-stimulation. This proved not to be the case $(p=0.44)$, indicating that the effect observed in the cathodal group was specific to stimulation over HG. The second analysis done for the cathodal-control group was designed to determine whether the reference electrode, functioning as the anode and placed supraorbitally, had any influence on pitch discrimination performance. The three levels of the factor "reference electrode condition" accordingly were "sham" (no current flowing, position

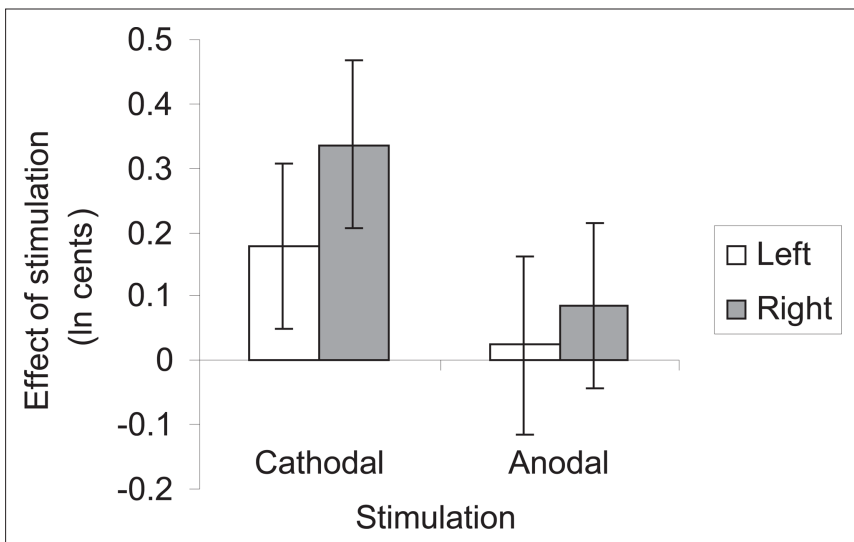

FIGURE 2 | Summary of results of ANCOVA. Bars represent mean subjects' performance after cathodal and anodal stimulation over left and right Heschl's gyrus, after correcting for learning/habituation effects. Error bars represent $95 \%$ confidence intervals. 


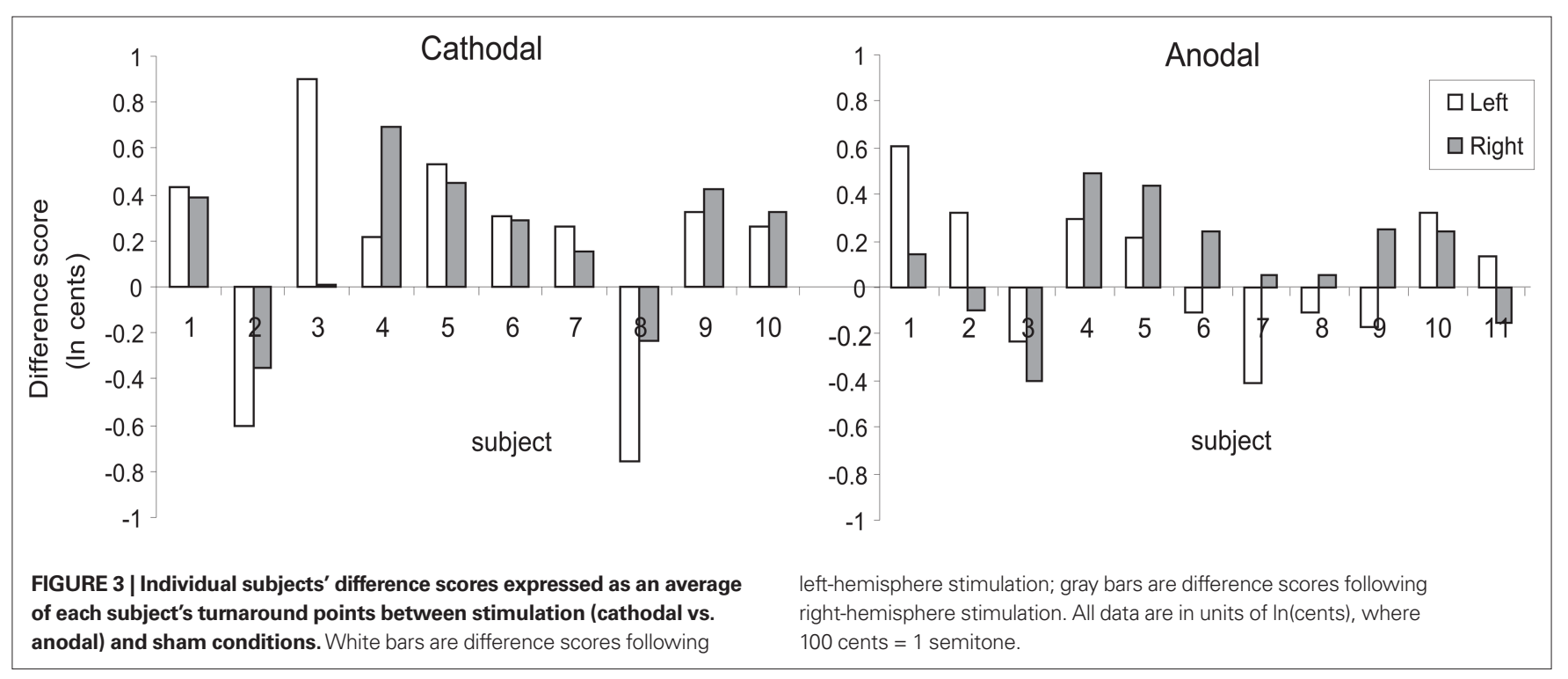

therefore irrelevant), "anodal, right," and "anodal, left.” This factor turned out not to be a significant source of variance $(p=0.66)$, indicating that the reference electrode presence in the supraorbital region did not influence pitch discrimination performance.

\section{ANODAL GROUP}

Anodal tDCS over left and right HG did not have any significant effect on pitch discrimination performance compared to sham $(p=0.39)$. As in the cathodal group, the main effects of subject and learning/habituation, and the main interaction of subject by learning/habituation, were all significant, indicating that subjects differed significantly both in baseline performance and in learning/ habituation $(p<0.05)$. However, none of the contrasts between stimulation conditions were significant $(p<0.05)$, suggesting that subjects were not affected by anodal stimulation.

\section{DISCUSSION}

Our results confirm the hypothesis that cathodal tDCS over the skull position of the first transverse gyrus of Heschl interferes with pitch direction discrimination on the left as well as on the right side compared to sham stimulation, with a stronger contribution of the right HG. This effect was not due to cathodal stimulation in general, since in a control group with cathodal stimulation over the occipital lobes, we did not find a detriment in performance. We did not find an enhancing effect in pitch discrimination with anodal stimulation over the skull positions of the right and left HG.

Although our results provide causal evidence for the involvement of the left and right HG in pitch direction discrimination, our results should be also be interpreted in light of the limitations of tDCS considering the uncertainty with regard to the focality of the stimulation, especially when the targeted brain region is internal, rather than a surface structure that is close to the skull. While our targeted stimulation sites were carefully chosen by using MRI to localize the exact brain region of interest, it is possible that the cortical region whose excitability was downregulated by the stimulation may have extended beyond HG and included adjacent parabelt regions of the auditory cortex in the superior temporal gyrus such as anterior parts of the planum temporale and/or posterior parts of the planum polare (Galaburda and Sanides, 1980; Formisano et al., 2003; Tramo et al., 2005). Specifically, the tDCS may have affected interneurons in these belt/parabelt regions that extend in a lateral-to-mesial direction, or it may have affected the lateral part of the primary auditory cortex, which extends along Heschl's gyrus with higher frequencies in the mesial direction and lower frequencies in the lateral direction (Lauter et al., 1985). In that regard, the choice of our relatively low center frequency of $200 \mathrm{~Hz}$ for the pitch direction discrimination task utilizes the lateral Heschl's gyrus, thus increasing the sensitivity of the functionally relevant lateral Heschl's region toward influence from tDCS applied on the lateral surface of the scalp.

Despite the fact that the left hemisphere is substantially involved in pitch discrimination, its contribution seems to be smaller or more easily compensated for than that of the right hemisphere, as evidenced by the stronger effect we found with cathodal stimulation over the right HG. This agrees with several lesion and imaging studies (Zatorre, 1988; Peretz, 1990; Zatorre et al., 1994; Johnsrude et al., 2000; Patterson et al., 2002; Tramo et al., 2005; Hyde et al., 2008; Okamoto et al., 2009) that suggest a right-hemisphere dominance for the processing of pitch-related stimuli. Nevertheless, our present results do not allow the conclusion that pitch discrimination is solely a "right-hemisphere function." As they show that the contribution of the left hemisphere is also significant. It is also important to note that our task was a pitch direction discrimination task. When Johnsrude et al. (2000) compared patients with lesions in $\mathrm{HG}$ to normal controls, they found no difference in pitch difference discrimination for lesions in right HG, but a significant impairment with respect to pitch direction discrimination, while no differences were found in either task for lesions in left HG. This might indicate an asymmetry that is confined to pitch direction discrimination but does not exist for pitch difference discrimination. On the other hand, in a study analyzing the effects of hemispheric lesions, Schuppert et al. (2000) used a pitch difference discrimination task to compare brain-lesioned patients to normal controls and found a greater detriment in performance for patients with lesions 
on the right than on the left side. Due to their small sample size, these differences did not reach significance, but they give an indication that a similar asymmetry might be present for pitch difference discrimination as for pitch direction discrimination.

The hypothesis that anodal stimulation over the skull position of right and left HG would lead to an enhancement of pitch discrimination could not be confirmed. Although the underlying processes that might lead to such an enhancement are not fully understood, it is suggested that anodal stimulation might increase the likelihood for plasticity and might influence learning processes by affecting NMDA receptor activity (Liebetanz et al., 2002) and ultimately long-term potentiation. This facilitation of learning might be the result of homeostatic plasticity processes whereby neuronal networks keep activity within a physiological range (Nitsche et al., 2007). Anodal stimulation would then enhance learning because the neural network is more excitable after stimulation owing to a tendency to return to an activity level experienced under anodal stimulation. Notably, improvement after anodal stimulation has mostly been observed with sensory and motor tasks for which subjects evidently had considerable room for improvement (Nitsche et al., 2003; Vines et al., 2006a, 2008; Ragert et al., 2008). In contrast, subjects may already be performing at ceiling levels in pitch discrimination, as psychophysical studies have indicated that pitch discrimination is a very sensitive perceptual ability in most humans. The sensitivity of this perceptual ability can be quantified as the fraction of stimulus intensity that has to be added to produce a just noticeable difference (the Weber fraction). This Weber fraction is about $1 \%$ for pitch direction discrimination, corresponding to 17 cents, for normal non-musically trained control subjects (Tramo et al., 2002). There are scarcely any other human sensory abilities that reach this level of sensitivity, i.e., have such a small Weber fraction. For instance, Baird and Noma (1978) report Weber fractions for different continua, such as light, sound, and weight that range from $2 \%$ (finger span, color saturation) to 24\% (smell). It might therefore not be possible to enhance pitch direction discrimination above its already very high baseline level by upregulating the excitability of the neurons that support it via tDCS. Furthermore, since our subjects already performed at a relatively high level, it might not have been possible to enhance performance further - at least

\section{REFERENCES}

Antal, A., Nitsche, M. A., and Paulus, W. (2001). External modulation of visual perception in humans. Neuroreport 12, 3553-3555.

Baird, J. C., and Noma, E. (1978). Fundamentals of Scaling and Psychophysics. New York: John Wiley \& Sons.

Baudewig, J., Nitsche, M. A., Paulus, W., and Frahm, J. (2001). Regional modulation of BOLD MRI responses to human sensorimotor activation by transcranial direct current stimulation. Magn. Reson. Med. 45, 196-201.

Chen, R., Classen, J., Gerloff, C., Celnik, P., Wassermann, E. M., Hallett, M., and Cohen, L. G. (1997). Depression of motor cortex excitability by low-frequency transcranial magnetic stimulation. Neurology 48, 1398-1403.

Formisano, E., Kim, D. S., Di Salle, F., van de Moortele, P. F., Ugurbil, K., and Goebel, R. (2003). Mirrorsymmetric tonotopic maps in human primary auditory cortex. Neuron 40, 859-869.

Gaab, N., Gaser, C., and Schlaug, G. (2006). Improvement-related functional plasticity following pitch memory training. Neuroimage 31, 255-263.

Gaab, N., Gaser, C., Zaehle, T., Jancke, L., and Schlaug, G. (2003). Functional anatomy of pitch memory - an fMRI study with sparse temporal sampling. Neuroimage 19, 1417-1426.

Gaab, N., and Schlaug, G. (2003). The effect of musicianship on pitch

not with short-term stimulation. This would agree with findings by others (Ziemann et al., 2004), who in a motor-learning context found that long-term potentiation (LTP)-like plasticity is difficult to induce in highly trained areas of the human cortex.

The existing literature on tDCS has also shown that it is more likely to induce a performance detriment than to enhance performance (Antal et al., 2001; Baudewig et al., 2001). Rogalewski et al. (2004) applied tDCS over the somatosensory cortex and found the same pattern as we did: cathodal stimulation disrupted tactile perception, while anodal stimulation had no effect. Another possibility is that the enhancing effect of the increased excitability in HG was counteracted by the effect of the virtual cathodes that arise in the vicinity of the anode (Roth, 1994; Nitsche et al., 2003). When a neuron is exposed to an electric field that does not affect its total extent, this leads to a polarization of the neuron as if it were stimulated not only where the field is present but also, in those areas unaffected by the real field, by a virtual field of opposite sign. Anodal stimulation of HG could in this way have had the side effect of an additional slight cathodal stimulation of surrounding fields which are also involved in pitch discrimination (Tramo et al., 2005).

In summary, this study showed that it is possible to interfere with performance in a pitch direction discrimination task through non-invasive brain stimulation over the skull position of the first transverse gyrus of Heschl that has been shown to lead to a downregulation in neural excitability. This effect was observed with stimulation over the left as well as right $\mathrm{HG}$ region, with the right side showing a somewhat stronger effect than the left. This effect could not be explained by cathodal stimulation in other parts of the brain and was not reversed by changing the current to anodal stimulation. By modulating the excitability of the auditory cortex via noninvasive brain stimulation, the present study provides a novel reverse-engineering approach that can be administered in vivo and combined with classical psychophysical methods to address the problem of pitch perception in the human brain.

\section{ACKNOWLEDGMENTS}

We gratefully acknowledge grant support from NIH (1RO1 DC008796, 3R01DC008796-02S1, R01 DC009823-01) and CIMIT.

memory in performance matched groups. Neuroreport 14, 2291-2295.

Galaburda, A., and Sanides, F. (1980). Cytoarchitectonic organization of the human auditory cortex. J. Comp. Neurol. 190, 597-610.

Griffiths, T. D., Johnsrude, I., Dean, J. L., and Green, G. G. (1999). A common neural substrate for the analysis of pitch and duration pattern in segmented sound? Neuroreport 10, 3825-3830.

Hickok, G., and Poeppel, D. (2007) The cortical organization of speech processing. Nat. Rev. Neurosci. 8, 393-402.

Hyde, K. L., Peretz, I., and Zatorre, R. J. (2008). Evidence for the role of the right auditory cortex in fine pitch resolution. Neuropsychologia 46, 632-639.

Johnsrude, I. S., Penhune, V. B., and Zatorre, R. J. (2000). Functional specificity in the right human auditory cortex for perceiving pitch direction. Brain 123(Pt 1), 155-163.

Langers, D. R., Backes, W.H., and van Dijk, P. (2007). Representation of lateralization and tonotopy in primary versus secondary human auditory cortex. Neuroimage 34, 264-273.

Lauter, J. L., Herscovitch, P., Formby, C., and Raichle, M. E. (1985). Tonotopic organization in human auditory cortex revealed by positron emission tomography. Hear. Res. 20, 199-205.

Liebetanz,D., Nitsche,M.A., Tergau, F., and Paulus, W. (2002). Pharmacological 
approach to the mechanisms of transcranial DC-stimulation-induced after-effects of human motor cortex excitability. Brain 125, 2238-2247.

Loui,P.,Li,H.C.,Hohmann,A., and Schlaug, G. (2010). Enhanced cortical connectivity in absolute pitch musicians: a model for local hyperconnectivity. J. Cogn. Neurosci.doi: 10.1162/jocn.2010.21500 [Epub ahead of print].

Micheyl, C., Delhommeau, K., Perrot, X., and Oxenham, A. J. (2006). Influence of musical and psychoacoustical training on pitch discrimination. Hear. Res. 219, 36-47.

Nitsche, M. A., and Paulus, W. (2000). Excitability changes induced in the human motor cortex by weak transcranial direct current stimulation. J. Physiol. 527(Pt 3), 633-639.

Nitsche, M. A., and Paulus, W. (2001). Sustained excitability elevations induced by transcranial DC motor cortex stimulation in humans. Neurology 57, 1899-1901.

Nitsche, M. A., Roth, A., Kuo, M. F., Fischer, A. K., Liebetanz, D., Lang, N., Tergau, F., and Paulus, W. (2007). Timing-dependent modulation of associative plasticity by general network excitability in the human motor cortex. J. Neurosci. 27, 3807-3812.

Nitsche, M. A., Schauenburg, A., Lang, N., Liebetanz, D., Exner, C., Paulus, W., and Tergau, F. (2003). Facilitation of implicit motor learning by weak transcranial direct current stimulation of the primary motor cortex in the human. $J$. Cogn. Neurosci. 15, 619-626.

Ohnishi, T., Matsuda, H.,Asada, T., Aruga, M., Hirakata, M., Nishikawa, M., Katoh, A., and Imabayashi, E. (2001). Functional anatomy of musical perception in musicians. Cereb. Cortex 11, 754-760.

Okamoto, H., Stracke, H., Draganova, R., and Pantev, C. (2009). Hemispheric asymmetry of auditory evoked fields elicited by spectral versus temporal stimulus change. Cereb. Cortex 19, 2290-2297.

Oldfield, R.C., (1971). The assessment and analysis of handedness: the Edinburgh inventory. Neuropsychologia 9, 97-113.

Pantev, C., Hoke, M., Lutkenhoner, B., and Lehnertz, K. (1989). Tonotopic organization of the auditory cortex: pitch versus frequency representation. Science New York, NY 246, 486-488.

Patterson, R. D., Uppenkamp, S., Johnsrude, I. S., and Griffiths, T. D. (2002). The processing of temporal pitch and melody information in auditory cortex. Neuron 36, 767-776.

Peretz, I., (1990). Processing of local and global musical information by unilateral brain-damaged patients. Brain 113(Pt 4), 1185-1205.

Platel, H., Price, C., Baron, J. C., Wise, R., Lambert, J., Frackowiak, R. S., Lechevalier, B., and Eustache, F. (1997). The structural components of music perception. A functional anatomical study. Brain 120(Pt 2), 229-243.

Ragert, P., Vandermeeren, Y., Camus, M., and Cohen, L. G. (2008). Improvement of spatial tactile acuity by transcranial direct current stimulation. Clin Neurophysiol 119, 805-811.

Rogalewski, A., Breitenstein, C., Nitsche, M. A., Paulus, W., and Knecht, S. (2004). Transcranial direct current stimulation disrupts tactile perception. Eur. J. Neurosci. 20, 313-316.

Roth, B. J., (1994). Mechanisms for electrical stimulation of excitable tissue. Crit. Rev. Biomed. Eng. 22, 253-305.

Schulze, K., Gaab, N., and Schlaug, G. (2009). Perceiving pitch absolutely: comparing absolute and relative pitch possessors in a pitch memory task. BMC Neurosci. 10, 106.

Schuppert, M., and Altenmuller, E. (1999) [Occupation-specific illnesses in musicians]. Versicherungsmedizin 51, 173-179.
Schuppert, M., Munte, T. F., Wieringa, B. M., and Altenmuller, E. (2000). Receptive amusia: evidence for crosshemispheric neural networks underlying music processing strategies. Brain 123(Pt 3), 546-559.

Scott, S. K., and Wise, R. J. (2004). The functional neuroanatomy of prelexical processing in speech perception. Cognition 92, 13-45.

Siebner, H. R., Lang, N., Rizzo, V., Nitsche, M. A., Paulus, W., Lemon, R. N., and Rothwell, J.C. (2004). Preconditioning of low-frequency repetitive transcranial magnetic stimulation with transcranial direct current stimulation evidence for homeostatic plasticity in the human motor cortex. J. Neurosci. 24, 3379-3385.

Talavage, T. M., Sereno, M. I., Melcher, J. R., Ledden, P. J., Rosen, B. R., and Dale, A. M. (2004). Tonotopic organization in human auditory cortex revealed by progressions of frequency sensitivity. $J$. Neurophysiol. 91, 1282-1296.

Tramo, M. J., Cariani, P. A., Koh, C. K. Makris, N., and Braida, L. D. (2005). Neurophysiology and neuroanatomy of pitch perception: auditory cortex. Ann. N. Y. Acad. Sci. 1060, 148-174.

Tramo, M. J., Shah, G. D., and Braida, L. D. (2002). Functional role of auditory cortex in frequency processing and pitch perception. J. Neurophysiol. 87, 122-139.

Vines, B. W., Cerruti, C., and Schlaug, G. (2008). Dual-hemisphere tDCS facilitates greater improvements for healthy subjects' non-dominant hand compared to uni-hemisphere stimulation. BMC Neurosci. 9, 103.

Vines, B. W., Nair, D. G., and Schlaug, G. (2006a). Contralateral and ipsilateral motor effects after transcranial direct current stimulation. Neuroreport 17, 671-674.

Vines, B. W., Schnider, N. M., and Schlaug, G. (2006b). Testing for causality with transcranial direct current stimulation: pitch memory and the left supramarginal gyrus. Neuroreport 17 1047-1050.

Zatorre, R. J. (1988). Pitch perception of complex tones and human temporallobe function. J. Acoust. Soc. Am. 84, 566-572.

Zatorre, R. J., Belin, P., and Penhune, V. B. (2002). Structure and function of auditory cortex: music and speech. Trends Cogn. Sci. 6, 37-46.

Zatorre, R. J., Evans, A. C., and Meyer, E. (1994). Neural mechanisms underlying melodic perception and memory for pitch. J. Neurosci. 14, 1908-1919.

Ziemann, U., Ilic, T. V., Pauli, C., Meintzschel, F., and Ruge, D. (2004). Learning modifies subsequent induction of long-term potentiation-like and long-term depression-like plasticity in human motor cortex. J. Neurosci. 24, 1666-1672.

Conflict of Interest Statement: The authors declare that the research was conducted in the absence of any commercial or financial relationships that could be construed as a potential conflict of interest.

Received: 18 July 2010; paper pending published: 30 September 2010; accepted: 20 October 2010; published online: 11 November 2010.

Citation: Mathys C, Loui P, Zheng $X$ and Schlaug G (2010) Non-invasive brain stimulation applied to Heschl's gyrus modulates pitch discrimination. Front. Psychology 1:193. doi: 10.3389/fpsyg.2010.00193

This article was submitted to Frontiers in Auditory Cognitive Neuroscience, a specialty of Frontiers in Psychology.

Copyright (C) 2010 Mathys, Loui, Zheng and Schlaug. This is an open-access article subject to an exclusive license agreement between the authors and the Frontiers Research Foundation, which permits unrestricted use, distribution, and reproduction in any medium, provided the original authors and source are credited. 